\title{
Estado nutricional y factores relacionados con Ia Parálisis Cerebral Infantil tipo cuadriparesia espástica severa en pacientes de 4 a 18 años del Centro de Rehabilitación Infantil Teletón Aguascalientes
}

Rodríguez-Hernández Al*, López-Olivares AL ${ }^{* *}$, Piña-Avilés CE ${ }^{* * *}$, Galván-Guerra M**, Ruvalcaba-Ledezma JC $C^{* * *}$

\section{Resumen \\ - La parálisis cerebral infantil es un trastorno del tono postural y del movimiento, de caracter persistente (pero no invariable), secundario a una agresión no progresiva - a un cerebro inmaduro. La relación entre Parálisis Cere- - bral Infantil (PCI) y la desnutrición es conocida, aunque - se tiene poca información de la frecuencia en países en - desarrollo, donde se añade el factor de la pobreza. En - este estudio describimos el estado nutricio, y factores - socioeconómicos relacionados con el mismo, en niños - con PCl espástica severa de entre 4 y 18 años de edad - que acuden al CRIT Aguascalientes. Encontramos que - el $67.3 \%$ padece malnutrición. El 50\% de la pobla-}

ción estudiada tiene algún padecimiento asociado a la PCl. El $82.7 \%$ es alimentado vía oral. En $94.2 \%$ de los casos, el cuidador principal es la madre y el grado de escolaridad máximo alcanzado por el cuidador fue secundaria en 38.5\%; finalmente, $51.9 \%$ presenta un nivel socioeconómico de pobreza (nivel socioeconómico según la encuesta nacional de ingresos y gastos, IMAE). Este estudio demostró que más de la mitad de los niños con $\mathrm{PCl}$ padecen un problema de malnutrición, patologías asociadas, limitaciones para la alimentación, nivel socioeconómico, escolaridad y ambiente familiar desfavorable o regular, que impactan de manera notable su estado nutricio. LUXMEDICA 5(I5): 3-8

Palabras clave: nutrición, $\mathrm{PCl}$, espasticidad

* Maestro en nutrición clínica. Centro de Rehabilitación Infantil Teletón Ags.

** Estudiante de maestría en Nutrición Clínica. Centro de Rehabilitación Infantil Teletón Ags.

*** Médicos especialistas. Centro de Rehabilitación Infantil Teletón Ags.

**** Doctor en Salud Pública. UNIVA-UdeG

Fecha de recibido: 12-abril-2010

Fecha de aceptado: 12-agosto-2010

Correspondencia: Rodríguez Hernández Al, Centro de Rehabilitación Infantil Teletón Ags.

Heroico colegio militar y Vázquez. Col. Santa Anita 2a. sección, Ags. Ags. adan_israellll@yahoo.com.mx 


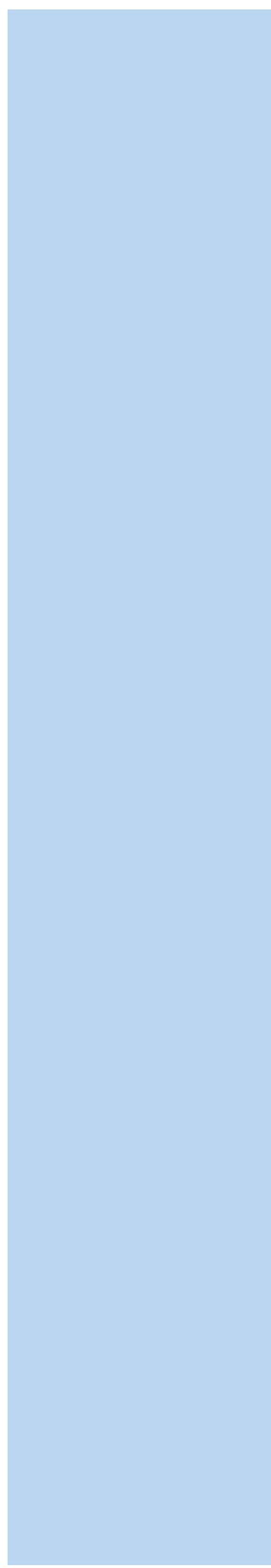

\section{Introducción}

La parálisis cerebral infantil $(\mathrm{PCl})$ describe un grupo de trastornos del desarrollo del movimiento y la postura, causantes de limitación de la actividad que se atribuyen a trastornos no progresivos que ocurrieron en el cerebro inmaduro ${ }^{1,2}$. La cifra de pacientes con $\mathrm{PCl}$ atendidos en el Centro de Rehabilitación Infantil Teletón Aguascalientes (CRIT) de agosto del 2008 a marzo del 2009 fue de 225, de los cuales 95 tienen una lesión severa $(42 \%)$. $^{3}$

Se estima que la prevalencia de la $\mathrm{PCl}$ es entre 2 y 3 casos por cada 1,000 nacidos vivos, siendo más alta en los países no industrializados. Considerando la tasa anual de natalidad en México, esto representa aproximadamente 1,000 casos nuevos al año en nuestro país ${ }^{2,3}$. En el Centro de Rehabilitación Infantil Teletón (CRIT) Aguascalientes los pacientes con diagnóstico de $\mathrm{PCl}$ representan poco más del $35 \%$ de los pacientes atendidos. ${ }^{3}$

Respecto a las lesiones neurológicas de la $\mathrm{PCl}$, que afectan la función neuromuscular del sistema gastrointestinal y que pueden alterar el estado nutricio de los pacientes se encuentran: disfunción motora oral, disquinesia faringoesofágica, dismotilidad esofágica e intestinal, las cuales ocasionan dificultad para la apertura bucal, incoordinación en la succión, masticación, deglución, reflujo gastroesofágico y constipación, entre otros ${ }^{4,5}$. El estado nutricio también es afectado por las características de la alimentación, la persona que alimenta al niño, la integración de la familia, la presencia del padre, el ingreso socioeconómico y la escolaridad del cuidador. ${ }^{6-8}$

Aunque la relación entre $\mathrm{PCl}$ y desnutrición proteico-calórica es conocida ${ }^{9,10}$, se tiene poca información de la frecuencia en países en desarrollo, como el nuestro, donde se añade el contexto de pobre$\mathrm{za}$, que plantea un conjunto de limitaciones (ingresos bajos, falta de alimento, vivienda, empleo, acceso a servicios de salud, educación e incremento de enfermedades infecciosas), que disminuyen la capacidad de una respuesta adecuada de estas familias. ${ }^{7,9,11}$

Esta realidad, hace a los niños con PCI más vulnerables a la aparición de complicaciones, como la desnutrición y trastornos relacionados, todo lo cual incide directamente sobre su salud y calidad de vida ${ }^{9}$.

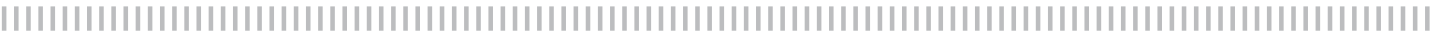

\section{Material y métodos}

Estudio observacional, descriptivo, transversal, retrospectivo. Se incluyeron a niños con $\mathrm{PCl}$ espástica severa, de entre 4 a 18 años del CRIT Aguascalientes, entre agosto 2008 y marzo de 2009. Se construyó un marco muestral, a partir del sistema electrónico de expedientes del CRIT Aguas- calientes y a partir del mismo, se realizó un muestreo aleatorio simple utilizando el programa Win Episcope 2.0 para calcular el tamaño de la muestra con un nivel de confianza del 95\%, margen de error 5\%, prevalencia esperada del $90 \%$, para una población de 95 sujetos y muestra total de 52 sujetos que representa el $54.7 \%$ de la 
totalidad de pacientes atendidos por esta patología en esta institución. Se revisaron los datos más recientes de la nota de rehabilitación pediátrica, obteniendo edad, sexo, y padecimientos asociados. Se revisaron las notas de integración social en base al estudio socioeconómico interno obteniendo los datos de: tipo de familia, ingresos económicos, cuidador principal y escolaridad de los padres. Se revisó la nota más reciente del servicio de nutrición para obtener el diagnóstico nutricio de los pacientes. Se entrevistó a la madre, bajo consentimiento informado, para obtener los datos restantes, consistencia de los alimentos, frecuencia y duración de las comidas, limitaciones motoras durante la alimentación a través una encuesta estructurada de nutrición y parálisis cerebral modificada por Nardi ${ }^{12}$. Para los últimos datos se aplicó el instrumento de medición de Méndez modificado, encuestando con previa carta de consentimiento informado. Una vez obtenida la información se vació en la hoja de captación de datos para su análisis utilizando estadística descriptiva paramétrica, con el programa SPSS 10.0 para Windows.

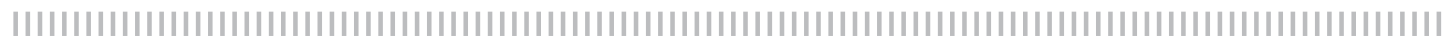

\section{Resultados}

De los 52 pacientes incluídos en nuestro estudio, encontramos que el $34.5 \%$ de los pacientes tienen desnutrición leve. En un porcentaje similar encontramos un estado nutricio normal y en menor proporción obesidad (3.8\%) como puede apreciarse en la figura 1. Asímismo el $9.6 \%$ de los pacientes fueron considerados con desnutrición crónica (afección de la talla por debajo del percentil 10).

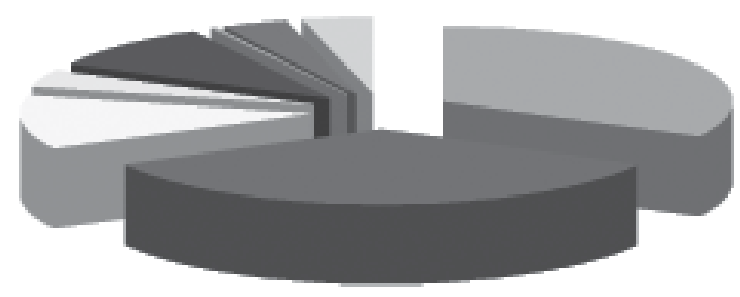

$32.7 \%$ Normalidad

$34.5 \%$ DN. Leve

$11.6 \%$ DN Moderada

$3.8 \%$ DN Grave marasmática

9.6\% DN Crónica

$0.2 \%$ DN Aguda

$3.8 \%$ Sobrepeso

$3.8 \%$ Obesidad

Figura 1. Distribución del estado nutricional en 52 pacientes con PCI tipo espástica severa del CRIT de Aguascalientes, agosto 2008-marzo2009 
Sobre las características socioeconómicas encontramos que más de la mitad de los pacientes pertenecen a familias con percepciones económicas entre 2900 y 5999 pesos mensuales (dato no mostrado), el promedio de escolaridad de los cuidadores fue de educación secundaria.

Entre los padecimientos asociados a la $\mathrm{PCl}$ los más prevalentes fueron el estreñimiento y la disfagia, pero encontramos también epilepsia, como lo observamos en la figura 2.

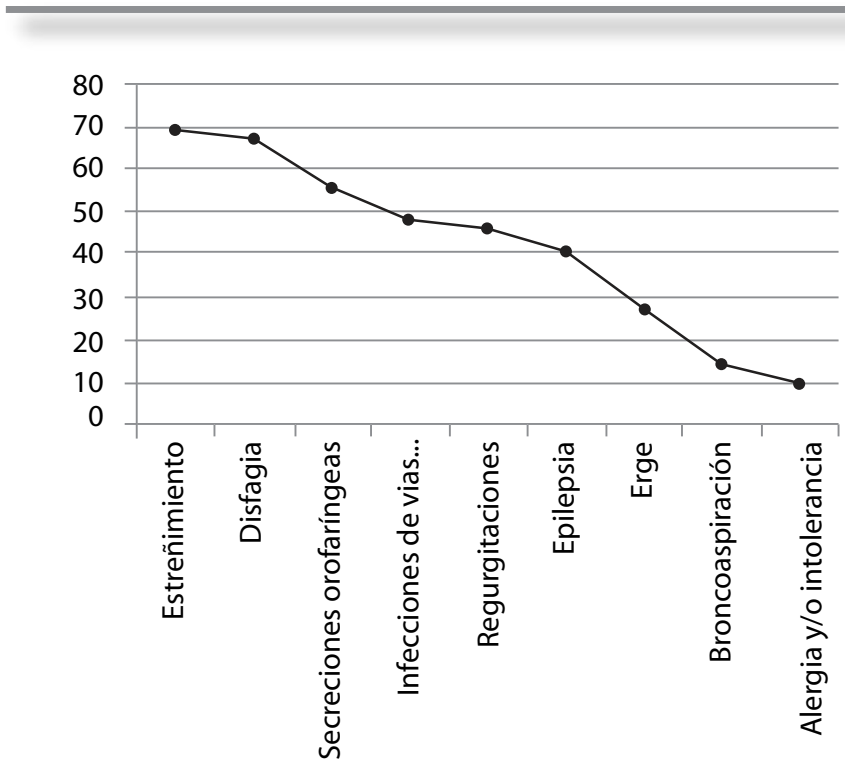

Figura 2. Se muestra por orden de frecuencia las alteraciones neurológicas que afectan el tracto gastrointestinal y otros sistemas en pacientes con PCI tipo espástica severa en el CRIT Aguascalientes.

En cuanto a las limitantes para la alimentación, encontramos que en la mayoría de seguida de otros factores como protrusión los pacientes tienen una mala masticación, (figura 3).

Limitantes para la alimentación

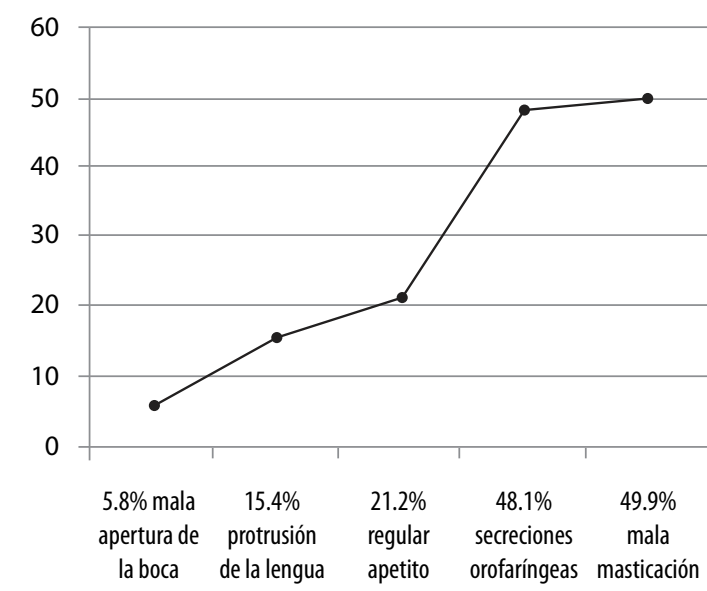

Figura 3. Factores que influyen como limitantes para la alimentación en 52 pacientes con $P C I$ espástica severa del CRIT Aguascalientes. 
Sobre las características del alimento, informadas por los cuidadores de estos pacientes, encontramos que la mayoría de ellos la reciben en forma de papilla $(55.8 \%)$, el $59.6 \%$ recibe complementos y/o suplementos alimenticios, y el $56 \%$ de ellos reciben 4 a 5 veces al día el alimento. La vía vía de alimentación es oral en el $82.7 \%$ y en el resto por gastrostomía. El
$17.3 \%$ de los cuidadores refieren que invierten más de 45 minutos para la alimentación del paciente.

Por último, encontramos que el cuidador principal de estos pacientes es la madre, auxiliado en un alto porcentaje (86.5\%) por el padre de familia. La mayoría de las familias de los pacientes corresponde al tipo nuclear $(80.6 \%)$.

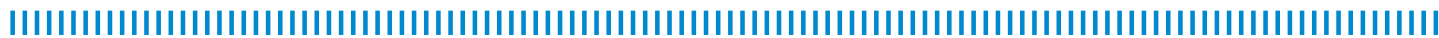

\section{Discusión}

La presente investigación tuvo como objetivo conocer el estado nutricional y los diversos factores patológicos y socioeconómicos relacionados, en niños con parálisis cerebral infantil tipo cuadriparesia espástica severa.

Nosotros encontramos, al igual que Águila y cols, que las familias de los niños con $\mathrm{PCl}$ son más vulnerables debido a factores socioeconómicos como la baja escolaridad del cuidador, los bajos ingresos familiares, malas condiciones de vivienda y padecimientos infecciones asociados a la enfermedad.

La escolaridad promedio en las madres de nuestros pacientes fue de secundaria lo que se ve estrechamente ligado al grado de desnutrición como lo refieren algunos autores, $8,13,14$ a mejor educación formal, mayor calidad de vida, lo que para Vial ${ }^{15}$ también fue de trascendencia, añadiendo que el alcoholismo en el padre y la situación en la pareja eran determinantes para la recuperación nutricional en los infantes.

También encontramos que en la mayoría de los casos la madre es el cuidador principal con el respaldo del padre de familia y solo en un $13.5 \%$ de los casos no se cuenta con la presencia del mismo.

Otro hallazgo en relación al estado nutricio, fue la diferencia entre los tipos de desnutrición según la temporalidad donde, en este estudio, la desnutrición crónica fue del $9.6 \%$ mientras que Águila 9 encontró un $43.5 \%$. Nosotros consideramos que la diferencia radica en que empleamos tablas de talla para la edad reajustadas por Stevenson $^{16}$ para las diferentes capacidades motoras de los niños con algún tipo de PC y que fueron publicadas en el 2006, dos años posteriores a la fecha en que fueron realizados los estudios de Águila y cols.

En cuanto a la desnutrición según severidad se encontró una prevalencia de $34.5 \%$ de desnutrición leve, seguido de $32.7 \%$ con diagnóstico de normalidad. Por otra parte Vásquez ${ }^{17}$ en su estudio encontró que un $41.2 \%$ de niños con $\mathrm{PCl}$ del Centro de Rehabilitación Teletón Occidente padecía algún tipo de obesidad, congruente con Beauquis ${ }^{7}$ donde evaluaron sujetos de varias discapacidades de quienes en más del $35 \%$ de la población estudiada, se encontró sobrepeso u obesidad. En este estudio encontramos que la población de niños con $\mathrm{PCl}$, sólo en un $7.6 \%$ padece algún grado de sobrepeso u obesidad.

En cuanto a la frecuencia de alteraciones asociadas, se coincide con Aguila ${ }^{9}$ donde el estreñimiento fue de $56 \%$ y en este estudio de $69 \%$, y lo contrario a los resultados de la enfermedad de reflujo gastroesofágico de $88.1 \%$ contra un $28 \%$ del presente. Cabe señalar que la diferencia radica en que las familias de la población no se han descartado enfermedad por reflujo.

Dentro de los padecimientos asociados y limitaciones para la alimentación se encontraron coincidencias con Nardi ${ }^{12}$ donde la mala apertura de boca fue de $23.3 \%$ y este estudio $5.8 \%$, regurgitaciones y/o vó- 
mitos de $32 \%$ contra el presente estudio de $40.5 \%$, infecciones de vías respiratorias de $64 \%$ contra $40.9 \%$, epilepsia $43.3 \%$ contra un $40 \%$.

Para los hallazgos sobre las características de la alimentación el consumo de alimentos tipo papilla, $\mathrm{Nardi}^{12}$ de $31.2 \%$ contra un $55.8 \%$, número comidas entre 4 y 5 por día $39.9 \%$ y este estudio el $55.4 \%$.

Un último dato es acerca de la vía de alimentación, donde la vía oral se presenta en un $82.7 \%$ en forma de papillas y solo un $17.3 \%$ tiene sonda por gastrostomía, lo que representa que las familias aún no tienen la cultura, aceptación o no cumplen con criterios de indicación de este tipo de alimentación, lo que en países desarrollados es lo contrario.

\section{Bibliografía}

1 Legido A, Katsetos CD. Parálisis cerebral: nuevos conceptos etiopatogénicos. Rev Neurol 2003; 36: 157 65.

2 Bax $M$, Goldstein $M$, Rosenbaum $P$, Leviton $A$, Paneth $\mathrm{N}$, Dan B, et al. Proposed definition and classification of cerebral palsy, April 2005. Dev Med Child Neurol 2005; 47: 571-6.

3 Gálvez Cisneros, Boletín estadístico y Epidemiológico 2008 del Centro de rehabilitación infantil teletón Aguascalientes 2009, Pag, 22:23.

4 Ramritu P, Finlayson K, Mitchell A, Croft G. Identification and nursing management of dysphagia in individuals with neurological impairment. The Joana Briggs Institute for evidence bases nursing 2000. no. 08

5 G. Pérez, J. Hernández. La disfagia su diagnóstico y tratamiento ¿Quién, cuándo y cómo? Revista Fac Med Univ Nac Coloma. 2004 vol. 52 no. 4

6 Dahl $M$, Thommessen $M$, Rasmussen $M$, Selberg $T$. Feeding and nutritional characteristics in children with moderate or severe cerebral palsy. Acta Pediátrica 1996; 85: 697-701

7 Beauquis Ana, Piombetti Laura, Problemas nutricionales y Factores asociados a los mismos en pacientes con discapacidad, Hospital General de Agudos "P. Piñero" Buenos Aires Argentina 2004.

8 Miren Lagonell, Gonzáles America, Escolaridad materna y desnutrición del hijo o hija. Centro Clínico Nutricional Menca de Leoni. Anales Venezuela Nutr v.18 n.2 Caracas dic. 2005 Disponible en: http://www. scielo.org.ve

9 Del Aguila A, Áibar P. Características nutricionales de niños con parálisis cerebral. ARIE - Villa El Salvador, 2004,

10 Mariño Mariana, Rojas Yusmely, Manejo nutricional de patologías neurológicas Centro De Atención Nutricional
Consideramos que lo referido por Mariño y cols, ${ }^{10}$ puede ser un referente importante para el manejo nutricional y antropométrico de los pacientes con $\mathrm{PCl}$.

En base a nuestros resultados podemos concluir que más de la mitad de los niños con $\mathrm{PCl}$ estudiados por nosotros, padecen un problema de malnutrición.

Para la evaluación nutricional antropométrica y diagnóstico nutricio de niños con $\mathrm{PCl}$ consideramos importante tomar como referencia las tablas de Stevenson para talla/edad.

Como propuesta, queremos enfatizar la importancia del manejo multidisciplinario de especialistas en pediatría, genética, rehabilitación, neurología, psicología, trabajo social y en este caso en especial la nutrición clínica pediátrica.

Infantil Antímano (CANIA) Caracas Venezuela 2002

11 Moreno Villares, Galiano Segovia, Alimentación En El Paciente Con Parálisis Cerebral Acta Pediátrica Española, Vol. 59, N.O 1, 2001

12 Nardi Adriana, "Estado nutricional y factores condicionantes en niños con parálisis cerebral" Hospital Central "Antonio Ma. Pinedo" Trabajo presentado para obtener el titulo de "Especialista en Puericultura y Pediatría " Barquisimeto, Venezuela febrero 2001, disponible en Url: http://bibmed.ucla.edu.ve/Edocs_ bmucla/textocompleto/TWS342N372001.pdf

13 Clark SL, Hankins GD. Temporal and demographic trends in cerebral palsy -fact and fiction. Am J Obstet Gynecol 2003; 18: 628-33.

14 Reddihough DS, Collins KJ. The epidemiology and causes of cerebral palsy. Aust J Physiother 2003; 49: 7-12.

15 Margarita Vial R. I; J orge Alvear A. *; Carmen Artaza B. "Microambiente de la desnutrición marásmica" Rev. Chil. Pediatr. 62 (4); 268-272, 1991.

16 Samsong-Fang LJ, Stevenson RD. Identification of malnutrition in children with cerebral palsy: poor performance of weight-forheight centiles. Dev Med Child Neurol 2000; 42: 162-168.

17 Vasquez Garibay, Evaluación antropométrica de la recuperación nutricia en niños con $\mathrm{PCl}$ En: Curso de nutrición infantil. La nutrición de los niños con discapacidad neuro - músculo - esquelética. $11^{\circ}$ Congreso (15 Nov, 2007).

\section{Agradecimiento}

Mi más sincero agradecimiento al Centro de Rehabilitación Infantil Teletón, a las áreas de Enseñanza, Dirección y Subdirección Médica, quienes han apoyado y confiado en este trabajo de investigación y han visto crecer como profesionista con el esfuerzo, constancia, amor y dedicación. 